\title{
SHARP BOUNDS ON THE DISTRIBUTION OF THE HARDY-LITTLEWOOD MAXIMAL FUNCTION
}

\author{
DAVID BLACKWELL ${ }^{1}$ AND LESTER E. DUBINS ${ }^{2}$
}

1. Introduction and summary. Somewhat tangentially to a recent study [1], we happened to notice an inequality which supplements one of Hardy and Littlewood, [2, Theorem 7, p. 95].

For an integrable function $f$ defined on the open unit interval $(0,1)$, the average value of $f$ over a set $J$ of positive measure is $A(J)=(P(J))^{-1} \int_{J} f(t) d t$ where $P(J)$ denotes the Lebesgue measure of $J$. The maximal function $M=M[f]$ studied in [2] is obtained by letting $M(x)$ be the least upper bound of $A(J)$ over all intervals $J$ which contain $x$. Recall that the distribution $\mathrm{Pg}^{-1}$ of a real-valued measurable function $g$ is defined for Borel sets $B$ by $\left(P g^{-1}\right)(B)=P\left(g^{-1}(B)\right)$.

It turns out that there is a function $\hat{f}$ that has the same distribution as $f$, but does not otherwise depend on $f$, for which $\int_{0}^{1} \phi(M(x)) d x$ $\leqq \int_{0}^{1} \phi(\hat{M}(x)) d x$ for all monotone increasing functions $\phi$, where $\hat{M}=M[\hat{f}]$ is the maximal function corresponding to $\hat{f}$. Though there are usually many such $\hat{f}$ there is one which is essentially unique and natural. Namely, a symmetric rearrangement of $f$ is a function $s$ on $(0,1)$ with the following three properties: (i) $s$ is symmetric about $x=1 / 2$; (ii) $s$ is nondecreasing for $0<x<1 / 2$; (iii) $s$ has the same distribution as $f$. Let $\hat{M}$ denote the maximal function associated with $s$, that is, $\hat{M}=M[s]$.

We can now state our result.

THEOREM. For all bounded monotone increasing functions $\phi$,

$$
\int_{0}^{1} \phi(M(x)) d x \leqq \int_{0}^{1} \phi(\hat{M}(x)) d x .
$$

Of course, the boundedness of $\phi$ is not at all important. All that matters is that both integrals in (1) exist.

2. Three lemmas. Since the indefinite integral of an integrable function is continuous, the first lemma is an immediate consequence of a celebrated lemma of Riesz [3, p. 6].

Received by the editors March 23, 1962.

${ }^{1}$ This paper was prepared with the partial support of the Office of Naval Research (Nonr-222-43).

2 Prepared with the partial support of the National Science Foundation. Grant G-14648. 
LEMMA 1. Let $f$ be an integrable function defined on an open interval and let $L$ be the set of $x$ for which there exists $z>x$ such that $\int_{x}^{z} f>0$. Then $\int_{L} f \geqq 0$.

Lemma 2. Let $f$ be an integrable function defined on an open interval, let $S$ be the set theoretic union of all intervals $J$ for which $\int_{J} f>0$, and let $S^{-}$be the set of $x \in S$ for which $f(x)<0$. Then

$$
2 \int_{f>0} f+\int_{S^{-}} f \geqq 0 \text {. }
$$

Proof. Let $R$ be the set of $x$ for which there exists $z<x$ such that $\int_{2}^{x} f>0$. Then $\int_{R} f \geqq 0$ just as $\int_{L} f \geqq 0$ in accordance with Lemma 1 . It is easily verified that $S=R \cup L$. Let $T=R \cap L$, let $S^{+}$be the set of $x \in S$ for which $f(x)>0$, and compute thus.

$$
\begin{aligned}
0 & \leqq \int_{R} f+\int_{L} f=\int_{T} f+\int_{S} f \leqq \int_{S^{+}} f+\int_{S} f \\
& =2 \int_{S^{+}} f+\int_{S^{-}} f \leqq 2 \int_{f>0} f+\int_{S^{-}} f .
\end{aligned}
$$

This proves the lemma.

A subset $B$ of the domain of a real-valued function $g$ is critical (for $g$ ) if there exists some $t,-\infty \leqq t<\infty$ such that $B$ contains every $x$ for which $g(x)>t$ but no $x$ for which $g(x)<t$.

LEMмA 3. Let $g$, strictly negative and integrable, be defined on some measure space $(U, \mathcal{u}, u)$. Suppose that $\int_{E} g \geqq \int_{B} g$ where $B$ is critical for $g$ and $E \in \mathcal{u}$, and $B \in \mathcal{u}$. Then $u(E) \leqq u(B)$.

Proof. If $u(B)=0$, then $0 \geqq \int_{E} g \geqq \int_{B} g=0$, implies that $\int_{E} g=0$. Since $g<0, u(E)=0 \leqq u(B)$. We may therefore suppose that $u(B)>0$. Under this assumption, since $g<0$, any $t$ for which $B$ is critical is negative. At the other extreme, if the only $t$ that made $B$ critical were $t=-\infty$, then $B$ would be $U$ and $u(B)=u(U) \geqq u(E)$. Henceforth, suppose that there exists a $t,-\infty<t<0$, such that $B$ contains all $x$ for which $g(x)>t$ and no $x$ for which $g(x)<t$. Of course, $g \leqq t$ on $E-B$, and $g \geqq t$ on $B-E$. Therefore,

$$
\int_{E-B} g \leqq t u(E-B) ; \quad \int_{B-E} g \geqq t u(B-E) .
$$

Also,

$$
\int_{E-B} g=\int_{E} g-\int_{E \cap_{B}} g \geqq \int_{B} g-\int_{E \cap_{B}} g=\int_{B-E} g .
$$


Inequalities (4) and (5) imply that $t u(B-E) \leqq t u(E-B)$. Since $-\infty<t<0, u(B-E) \geqq u(E-B)$, which, of course, implies that $u(B) \geqq u(E)$. The proof of Lemma 3 is complete.

3. Proof of Theorem. Notice that $S$ as defined in Lemma 2 is the same as the set of $x$ for which $M(x)>0$. Let $S$ be the set of $x$ for which $\hat{M}(x)>0$. Our principal task remaining is to show that $P(S) \leqq P(\hat{S})$.

Setting aside the trivial cases in which $P(S)=0$ or $P(\hat{S})=1$, notice that $\hat{S}$ is an interval of length $<1$, symmetric about $1 / 2$, and is therefore a critical set for $s$. The defining property of $\hat{S}$ together with its symmetry about $1 / 2$ easily imply

$$
2 \int_{s>0} s+\int_{\hat{\mathrm{s}}^{-}} s=0
$$

Since $\int_{s>0} s=\int_{f>0} f,(6)$ and (2) imply

$$
\int_{S^{-}} f \geqq \int_{\hat{\mathrm{S}}^{-}} s .
$$

Since $s$ and $f$ have the same distribution, and $\hat{S}^{-}$is critical for $s$ restricted to the set where $s<0$, there exists a set $B$ on which $f<0$, critical for $f$, such that

$$
\int_{\hat{\mathrm{S}}^{-}} s=\int_{B} f, \text { and } P\left(\hat{S}^{-}\right)=P(B) .
$$

The first half of (8) together with (7) implies

$$
\int_{S^{-}} f \geqq \int_{B} f
$$

Apply Lemma 3 to (9) to conclude that $P\left(S^{-}\right) \leqq P(B)$, which, in view of the second half of (8), gives $P\left(S^{-}\right) \leqq P\left(\hat{S}^{-}\right)$. Then $P(S)$ $\leqq P(f \geqq 0)+P\left(S^{-}\right) \leqq P(s \geqq 0)+P\left(\hat{S}^{-}\right)=P(\hat{S})$. Replace $S$ by $M^{-1}(0, \infty)$ and $\hat{S}$ by $\hat{M}^{-1}(0, \infty)$, and conclude that $P M^{-1}(0, \infty) \leqq P \hat{M}^{-1}(0, \infty)$. To see that $P M^{-1}(t, \infty) \leqq P \hat{M}^{-1}(t, \infty)$ for all $t$, merely notice that the $s, M$, and $\hat{M}$ which correspond to $f-t$ are obtained from the $s, M$, and $\hat{M}$ which correspond to $f$ by subtracting the same constant $t$. Consequently (1) holds whenever $\phi$ has the form $\phi(r)=1$ for $r>t$ and $\phi(r)=0$ for $r \leqq t$. Since (1) is a linear inequality, it holds for finite nonnegative linear combinations of such $\phi$. Since any bounded monotone nondecreasing continuous $\phi$ can be uniformly approximated by such step functions, and since any bounded monotone nondecreasing $\phi$ is a pointwise limit of a uniformly bounded sequence of such con- 
tinuous $\phi$, the dominated convergence theorem of Lebesgue applies to complete the proof of the Theorem.

4. A Remark. The theorem above is of the same nature as the "one-sided" theorem, Theorem 5 in [2, p. 92]. Hardy and Littlewood deduce their theorem from its interesting discrete analogue [2, Theorem 2, p. 84]. Such a procedure was not open to us, for the discrete analogue of our theorem does not hold. Consider for example, the possible rearrangements of the finite sequence $1,2,4,8$, and verify that among their "two-sided" maximal functions none dominates all the others.

\section{REFERENCES}

1. D. Blackwell and L. E. Dubins, $A$ converse to the dominated convergence theorem, Illinois J. Math. (to appear).

2. G. H. Hardy and J. E. Littlewood, A maximal theorem with function-theoretic applications, Acta Math. 54 (1930), 81-116.

3. F. Riesz and Béla Sz-Nagy, Leçons d'analyse fonctionelle, 2nd ed., Academie des Sciences de Hongrie, Budapest, 1953.

University of California, Berkeley 